The longevity of Y chromosomes

\section{The Human Y chromosome is not dead (yet)}

$P$ de Knijff

Heredity (2006) 97, 377-378. doi:10.1038/sj.hdy.6800896; published online 4 October 2006

T here are those who want us to believe that, given sufficient time, the human Y-chromosome (HY) will disappear (Graves, 2006). Their reasoning is simple and straightforward. Owing to lack of recombination the HY has degenerated in size and number of genes during its 300 million years of splendid isolation and there is nothing to prevent this process from continuation towards its impending demise. Still, although there are many strong arguments in favor of this hypothesis, there might be some hope.

This hope is based on two different lines of research. First, the two recently published complete chimpanzee Y-chromosome sequences enables a direct and detailed comparison between the $\mathrm{Hu}$ man Y-chromosome and its evolutionary closest relative (Hughes et al., 2005; Kuroki et al., 2006). From this, it appears that at least during the last 6 million years of human evolution the HY did not suffer from any detectable loss of genes. This cross-species comparison also revealed strong signals of purifying selection acting on expressed genes on the HY. It is the second line of evidence, described in some more detail below, which could explain at least in part this unexpected escape from extinction.

Obviously, recombination is not the only genomic redistribution mechanism. A continuous process of gene conversion, homologous recombination, inversion, deletion, and duplication events, influenced by purifying selection and hitchhiking could be sufficiently powerful to prevent the $H Y$ from its ultimate fate (Hughes et al., 2005). If this is true, the remarkable structural repetitive nature of the HY would no longer be a little understood byproduct of evolution, but actually serve an important purpose.

In a recent report, Repping et al. (2006) describe in much detail the remarkable flexibility of the HY. Their approach, involving a lot of work, is very simple and makes clever use of the availability of a stable and reliable phylogeny of all extant HY (YCC-con- sortium, 2002). They carefully selected 47 cell lines, each representing a distinct branch of the HY-tree, and analyzed these samples for the possible presence of nine large-scale structural polymorphisms. All structural polymorphisms observed among the 47 samples were superimposed on the fully resolved HY-phylogeny (see Figure 1). This enabled the authors to estimate the minimum number of structural large-scale structural variants a daunting task. mutations, per polymorphism. Without the HY-phylogeny, this would have been an almost impossible task. As the available HY-phylogeny could be biased (not based on randomly discovered mutations), the authors resequenced $\sim 80 \mathrm{~kb}$ in all 47 samples and discovered 94 new Y-chromosome SNPs. These SNPs produced an independently and unbiased HY-phylogeny which was near identical to the old one. Four frequently polymorphic structural variants were detected; the distal Yq heterochromatin, the TSPY array, the IR3 inversion, and $\mathrm{AZFC}$, with lower bound mutation frequency estimate between 2.2 and $4.4 \times 10^{-4}$. These remarkable high mutation rates approaches those of microsatellites. Another interesting observation is the predominance of inversions in $\mathrm{AZFC}$, resulting in very few chromosomes with different gene copy numbers. This could be yet another confirmation of the hypothesis

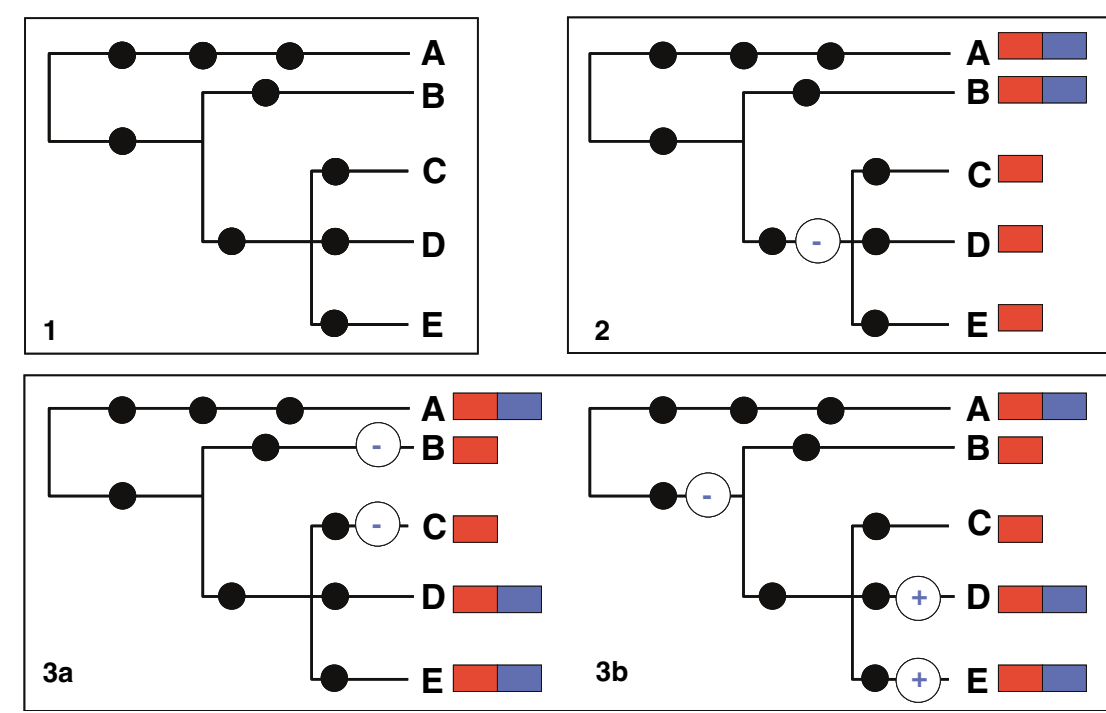

Figure 1 The power of a stable phylogeny of the human Y-chromosome. Panel 1 shows the phylogeny of five hypothetical Y-chromosomes A-E. This phylogeny is based on nine nonrecurrent mutations (indicated by black dots). The phylogeny is fully resolved. Chromosome $\mathrm{A}$ is the oldest, most ancestral Y-chromosome. Chromosomes C-E represents three recently derived Y-chromosomes. Panel 2 illustrates the screening results of a long-range repeat of each of the five Y-chromosomes. A and B have two copies of this repeat. C-E have only a single copy. This can be explained - assuming a minimal or most parsimonious mutation model - by a single deletion event (indicated by the encircled ' -') shortly before C, D, and E diverged from each other. Panels $3 a$ and $b$ illustrates a slightly more complex model. The deletion of a repeat in $\mathrm{B}$ and $\mathrm{C}$ can either be explained by two independent deletion events (3a) or a single deletion event, subsequently followed by two insertion events (indicated by an encircled ' + '). In this case, the model in (3a) (involving only two mutational events) is preferred above (3b) (involving three mutational events. From this kind of information, with some assumptions, one can easily estimate the mutation frequency (in number of mutations per generation) of such numerical polymorphisms (explained in detail in the supplementary information of Repping et al., 2006). As most of the Y-chromosome does not recombine, the fully resolved phylogeny of extant human Y-chromosomes can be seen as a genetic archive where the order in time of almost all mutation events is preserved. Estimating the number of recurrent mutations is thus relatively simple. On the autosomes, because of recombination, such phylogenies are never fully resolved. This makes estimating recurrent mutation rates of 
that natural selection has a prominent role in keeping extreme gene copy variation within acceptable limits. If true, this means that lack of recombination on the HY has been counterbalanced by similar efficient processes protecting this chromosome from accumulating deleterious variants. If this escape mechanism was sufficient protective for the last 6 million years, why not for ever?

Peter de Knijff is at the Department of Human Genetics, Leiden University Medical Center, PO Box 9600, 2300 RC Leiden, The Netherlands.
E-mail: P.de_Knijff@lumc.n

Graves JAM (2006). Cell 124: 901-914.

Hughes JF et al. (2005). Nature 437: 101-104.

Kuroki Y et al. (2006). Nat Genet 38: 158-167.

Repping S et al. (2006). Nat Genet 38: 463-467.

The Y chromosome consortium (2002). Genome Res 12: 339-348.

\section{Editor's suggested reading}

Charlesworth D, Charlesworth B, Marais G (2005) Steps in the evolution of heteromorphic sex chromosomes. Heredity 95: 118-128.
Filatov D (2005). Stickleback's view of sex chromosome evolution. Heredity 94: 275-276.

Lawson Handley LJ, Hammond RL, Emaresi G, Reber A, Perrin N (2006). Low Y chromosome variation in Saudi-Arabian hamadryas baboons (Papio hamadryas hamadryas). Heredity 96: 298-303.

Lim KY, Werlemark G, Matyasek R, Bringloe JB, Sieber V, El Mokadem H et al. (2005). Evolutionary implications of permanent odd polyploidy in the stable sexual, pentaploid of Rosa canina L. Heredity 94: 501-506. 